Reuri, F: C., Nicrenkrankheiten, Huber, Bern und Stuttgart (1960). - 14. Smith, H. W., J. Clin. Invest. 20, 631 (1941). 15. Richards, A. N., Proc. Roy. Soc. (London), Ser. B 126, 398 (1938). - 16. Ferguson, M. H., O. Olbrich, J. S. Robson und C. P. Stewart, Quart. J. exp. Physiol. 35, 251 (1950). - 17. KENNEDY, T. J. und J. K.EH, J. Clin. Invest. 32, 90 (1953). 18. FREY, J., Klin. Wschr. 36, 11 (1958). - 19. GAYER, J., Verh. Dtsch. Ges. inn. Med. 65, 599 (1959). - 20. Goldring, W. und H. Chasis, Hypertension and hypertensive disease, The Commonwealth Fund, New York (1944). - 21. BRUN, C., T. HiLden und F. RaAschou, Acta med. Scand. Suppl. 234, 71 (1949). 22. Stewart, G. N., Amer. J. Physiol. 57, 27 (1921). - 23. Frck, A., S. ber. Physik.-Med. Ges. Würzburg, 16 (1870). - 24. Reubi, F. C., N. Gossivetrer und R. Gürtler, Proc. Soc. exp. Biol. Med. 111, 760 (1962). - 25. SMrth, H. W., W. Goldring und H. CHiasrs, J. Mount Sinai Hosp. N. Y. 10, 59 (1943). - 26. DeETJEN, P., Persönliche Mitteilung. - 27. MALvin, R. L., W. S. WILDE und L. P. Sullivan, Amer. J. Physiol. 194, 135 (1958). — 28. Klein-
SCHMIdt, A. und S. HÄNZE, Erg. inn. Med. Khlkd. 14, 239 (1960). 29. Reubi, F. C., C. Vorburger und H. M. Keller, Clin. Sc., London 23, 2 (1962). - 30. Reubi, F. C., Helvet. med. acta, Suppl. 26 (1950). - 31. Goldring, W., H. Chasis, H. A. Ranges und H. W. SMrth, J. Clin. Invest. 19, 739 (1940). - 32. SMITH, H. W., W. Goldring und H. Chasis, J. Clin. Invest. 17, 263 (1938). 33. Bratton, A. C. und E. K. Marshall, J. biol. Chemistry, 128, 537 (1939). - 34. Gilaran, A., F. S. Philtps und E. S. Koelle, Amer. J. Physiol. 146, 348 (1946). - 35. Schireiner, G. E., Proc. Soc. exp. Biol. Med. 74, 117 (1950). - 36. EArLe jr., D. P., S. Sherry, L. W. Eichna und N. J. Conan, Amer. J. Med. 11, 283 (1951). - 37. BRADLEY, S.W., Factors regulating blood pressure, 1. Conf., S. 119, J. Macy Foundation, New York (1947). 38. Breed, E. S., M. H. Maxwell und H.W. S.irth, Amer. J. Med. 9, 216 (1950). - 39. WatKIN, D. M. und N. W'. Shock, J. Clin. Invest. 34, 969 (1955). - 40. Reubr, F. C. und C. VorBURGER, Nierenkrankheiten im Alter, Handbuch für praktische Geriatric (im Druck). Ferdinand Enke Verlag, Stuttgart.

Dr. med. C. Vorburger

Medizinische Poliklinik der Universität Bern Bern (Schweiz)

Freiburgstr. 3

\title{
Zur Bestimmung der Leucinaminopeptidase-Aktivität im Serum
}

\author{
Von \\ H. SCHÖN, B. RÄSSLER und H.-J. WEYERGRAF \\ Aus der Medizinischen Klinik der Universität Erlangen-Nürnberg (Direktor: Prof. Dr. N. Henning)
}

(Der Schriftleitung zugegangen am 25. Februar 1963)

\begin{abstract}
Es wird über eine eigene Modifikation der BR̈Atron-Marsflall-Reaktion zur Bestimmung der Leucinaminopeptidase-Aktivität im Blutserum berichtet. Normwerte sind für Männer 18,6-36,7 IE, für Frauen 16,3-29,2 IE. Eine Erhöhung der LAP-Aktivität fand sich bei Cholangitis, Cholecystitis, Lebermetastasen, Gravidität, Verschlußikterus, akuter Hepatitis, akuter Pankreatitis und Pankreas-Carcinom. Vergleichende Untersuchungen der LAP mit den Transaminasen (GPT und GOT) und der alkalischen Phosphatase lassen eine diagnostische Überlegenheit der LAP gegenüber der alkalischen Phosphatase beim Verschlußikterus, sowie bei extra- oder intrahepatisch bedingten Cholostasen gegenüber den Transaminasen möglich erscheinen. Die Verwertbarkeit der LAP in der Pankreasdiagnostik bedarf der weiteren Úberprüfung.
\end{abstract}

A modification of the BratToN-MrARSHALL reaction for the determination of leucine amino peptidase activity in blood serum is reported. Normal values are 18.6-36.7 IU for men, and 16.3-29.2 IU for women. There is an increased LAP activity in cholangitis, cholecystitis, liver metastases, pregnancy, obstructive jaundice, acute hepatitis, acute pancreatitis and pancreas carcinoma. Comparative studies with the transaminases (GPT and GOT) and the alkaline phosphatases show that LAP may be diagnostically superior to alkaline phosphatase for obstructive jaundice, and to transaminases for extra- or intrahepatic-determined cholostases. The applicability of LAP in pancreatic diagnoses requires further testing.

Im Jahre 1929 gelang LiNDERSTRøM (1) die Darstellung der Leucinaminopeptidase (LAP) im Darmsaft. Diese Exopeptidase unterscheidet sich von anderen Peptidasen dadurch, daß sie L-Leucylglycylglycin, L-Leucylglycin und L-Leucinamid hydrolysiert. Obwohl über ihre Bedeutung innerhalb des pflanzlichen und tierischen
Stoffwechsels noch keine Klarheit herrscht, spaltet sie wahrscheinlich bei Anwesenheit von Mangan- oder Magnesiumionen Peptidbindungen in Nachbarschaft eines L-Leucin-Restes $(2,3)$. - Über erhöhte Enzymaktivitäten im Serum wurde bei Leber-, Gallen- und Pankreaskrankheiten $(4,5,6,7,8)$, bei Gravidität (6) 
und Hauteffloreszenzen (9) berichtet. Dabei liegt der diagnostische Wert des LAP-Tests im Serum bisher auf dem Gebiet der Leber-, Gallen- und Pankreaskrankheiten.

In der vorliegenden Arbeit prüften wir mittels einer eigenen Modifikation der bisher benutzten BRATTONMARSHALL-Reaktion (10) die LAP-Aktivität im Serum von Normalpersonen, sowie von Patienten unserer Klinik. Besonders berücksichtigt wurden Leber- und Pankreaskrankheiten sowie extrahepatisch bedingte Gallenabflußbehinderungen durch Tumoren oder Konkremente. Außerdem sollte der diagnostische und prognostische Wert der LAP-Bestimmung gegenüber der bereits in die Enzymdiagnostik fest eingeführten Bestimmung der Transaminasen (GPT, GOT) und der alkalischen Phosphatase abgegrenzt werden.

\section{Methodik}

Prinzip: Die Bestimmung der LeucinaminopeptidaseAktivität im Serum wird durch die Spaltung von Leucyl$\beta$-naphthylamid und kolorimetrische Messung des freigesetzten $\beta$-Naphthylamins nach Eiweißfällung durch Diazotierung und Kupplung mit Naphthyläthylendiamin ermöglicht.

\section{Reagentien:}

1. $0,05 m$ Trispuffer, $\mathrm{pH}=7,2$. -2 . L-Leucyl- $\beta$-naphthylamidhydrochlorid*, 0,2 proz. methanol. Lösung. -3 . Perchlorsäure, p. a. 12,5 proz. 4. Natriumnitrit, p. a. 0,2 proz. wäßrige Lösung, frisch bereitet. - 5. Ammoniumsulfamat, p. a. 0,5 proz. wäßrige Lösung, frisch bereitet. - 6. N-(1-Naphthyl)-äthylendiamindihydrochlorid, p. a. methanol. Lösung $(0,5 \mathrm{mg} / \mathrm{ml})$.

Ausfübrung: $0,5 \mathrm{~m} l$ einer 20 proz. Serumverdünnung ( $1 \mathrm{~m} l$ Serum, ad $5 \mathrm{~m} l$ physiol. Kochsalzlösung) und $1,3 \mathrm{ml}$ Trispuffer werden mit $0,2 \mathrm{~m} /$ Substratlösung $30 \mathrm{Min}$. bei $37^{\circ} \mathrm{im}$ Wasserbad inkubiert. Anschließend wird die Fermentreaktion durch Zugabe von $1 \mathrm{ml}$ Perchlorsäure abgestoppt. Nach Zentrifugieren wird $1 \mathrm{ml}$ des Uberstandes mit $1 \mathrm{~m} l$ Nitritlösung im Wasserbad bei $37^{\circ} 10 \mathrm{Min}$. lang inkubiert, der Nitritüberschuß durch Zugabe von $1 \mathrm{ml}$ Sulfamatlösung zerstört und nach $2 \mathrm{Min} .2 \mathrm{ml}$ der Naphtyläthylendiaminlösung zugesetzt. Diese Lösung wird unter Lichtausschluß in verschlossenen Reagenzgläsern $30 \mathrm{Min}$. bei $37^{\circ}$ belassen, auf Zimmertemperatur abgekühlt, und die Extinktion gegen den Reagentienblindwert im Photometer bei $578 \mathrm{~m} \mu$ und $1 \mathrm{~cm}$ Schichtdicke gemessen. - Der Reagentienblindwert unterscheidet sich dadurch vom Meßwert, daß das verdünnte Serum erst nach der Perchlorsäure zugefügt wird. - Es werden nur solche Meßergebnisse verwendet, deren Extinktion zwischen 0,1 und 0,8 liegt. Bei höherer Aktivität der LAP muß das Serum weiter verdünnt werden. Zur Ermittlung der durch enzymatische Hydrolyse aus L-Leucyl- $\beta$-Naphthylamid freigesetzten $\beta$-Naphthylaminmenge dient eine Eichkurve, die unter Verwendung einer von $1-10 \mu \mathrm{g}$ ansteigenden $\beta$-Naphthylaminlösung erstellt wurde (Abbildung 1).

\section{Methodische Ergebnisse:}

Zum Nachweis der LAP-Aktivität im Blutserum wurde eine eigene Modifikation der BRATTON-MARSHALLReaktion benutzt, wodurch die Empfindlichkeit und Reproduzierbarkeit der Methode durch Anwendung

*) Mann Research Lab. Inc., New York.

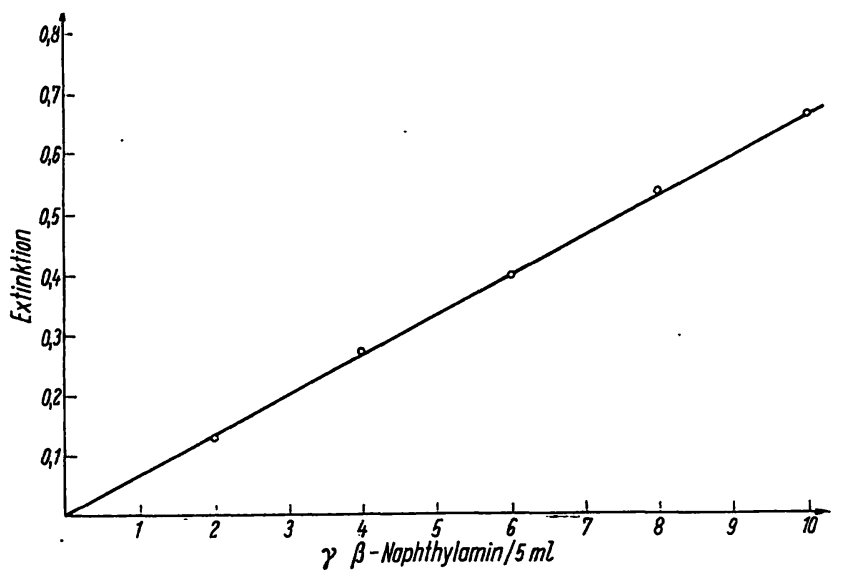

Abb. 1

Eichkurve; graphische Darstellung der Extinktion in Abhängigkeit von der $\beta$-Naphthylaminmenge (Filter $578 \mathrm{~m} \mu$, Schicht $1 \mathrm{~cm}$, Photometer Eppendorf)

optimaler Reaktionsbedingungen wesentlich gesteigert werden konnte. So erhielten wir im Gegensatz zu Goldbarg (6) u. a. $(11,8)$, erst nach 10 Min. dauernder Diazotierung mit 0,2 proz. $\mathrm{NaNO}_{2}$-Lösung bei $37^{\circ}$ unter Lichtausschluß einen Endwert, der auch bei Temperaturerhöhung auf $40^{\circ}$ und verlängerter Einwirkungszeit auf $30 \mathrm{Min}$. unverändert blieb. Die Dauer der Kupplungsreaktion wird von GreEN (11) mit 2 Stdn., von Goldbarg (6) nur mit $15 \mathrm{Min}$. angegeben. Wir erhielten nach $30 \mathrm{Min}$. Inkubation bei $37^{\circ}$ einen maximalen, über mehrere Stunden stabilen Extinktionswert. Nach Ansicht verschiedener Autoren ist die LAP-Aktivität an die Anwesenheit von $\mathrm{Mn}^{++}$- und $\mathrm{Mg}^{++}$-Ionen gebunden $(6,8)$, was unsere Versuchsergebnisse bestätigen. - Das Wirkungsoptimum des Ferments liegt nach unserer Feststellung im schwach alkalischen Bereich bei $\mathrm{pH}=7,2-7,3$. Diese Ergebnisse finden ihre Bestätigung bei GoldDARG (6) und GREEN (11), während BRAUN-FALCO und SALFELDT (12) ihre Versuche im pHBereich 7,4-7,8 durchführten. Der Meßwert erfährt bei Verwendung verschiedener Puffersubstanzen, wie Veronal, Tris- oder Phosphatpuffer keine Änderung $(6,13$, 12). - Die 20 proz. Serumverdünnung $(1 \mathrm{~m} l$ Serum, ad $5 \mathrm{ml}$ aqua bidest.) ergibt eine in allen Extinktionswerten optimale Fermentaktivität. Die graphische Darstellung zeigt eine Gerade, während bei höheren Serumkonzentrationen die Enzymaktivität der vorgelegten Serummenge nicht direkt proportional ist. Die auch von anderen Autoren $(12,3)$ festgestellte Abweichung von der Linearen bei höheren Serumkonzentrationen kann sowohl auf einer Störung der Nachweisreaktion (Eiweißadsorption des $\beta$-Naphthylamin), als auch auf einer Beeinflussung der Fermentaktivität (Ausschaltung eines Inaktivators durch Verdünnung, zu geringe Substratkonzentration) beruhen. Der Vorteil der BrattonMARSHALI-Reaktion liegt gerade darin, daß die Eiweißadsorption im Gegensatz zum Nachweis mit Echtblausalz (direkte Kupplung) kaum ins Gewicht fällt (Abbildung 2). 


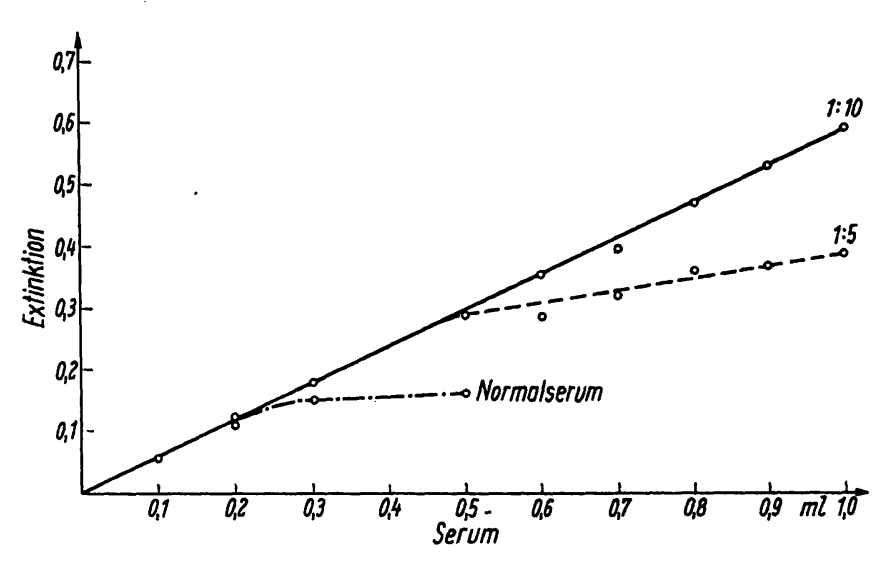

Abb. 2

Serumkonzentrationskurve; Extinktion in Abhängigkeit von der Serummenge (Serumverdünnung 1:5;1:10; Normalserum, meßtechn. Daten s. Abbildung 1)

Weiter untersuchten wir Testansätze mit verschiedenen Substratkonzentrationen und fanden, $\mathrm{da} \beta 0,2 \mathrm{~m} l$ einer $200 \mathrm{mg}$ proz. Substratlösung ( $=400 \mu \mathrm{g}$ im Ansatz) von L-Leucyl- $\beta$-Naphthylamidhydrochlorid zur reproduzierbaren Erfassung der Fermentaktivität im Serum auch bei hochpathologischen Werten voll ausreicht (Abbildung 3 ).

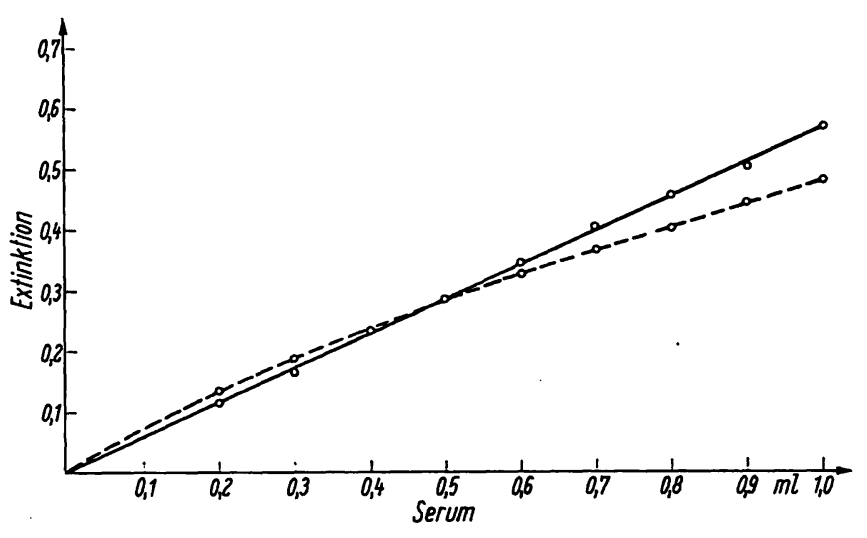

Abb. 3

Substratkonzentrationsprüfung in Abhängigkeit von der Enzymaktivität (angezogene Kurve $200 \mathrm{mg} / 100 \mathrm{ml}$, gestrichelte Kurve $100 \mathrm{mg} / 100 \mathrm{~m} l$ Substrat). Meßtechn. Daten s. Abbildung 1

Außerdem wurde die Fermentaktivität verschiedener 20 proz. Serumverdünnungen bei konstanter Substratkonzentration über eine Inkubationszeit von $100 \mathrm{Min}$. geprüft. Dabei zeigte sich, daß der Anstieg der einzelnen Kurven pro Zeiteinheit linear ist (Abbildung 4).

- Dennoch entschlossen wir uns, die Inkubationszeit so kurz wie möglich zu halten, um den zeitlichen Erfordernissen der Routinemethodik zu entsprechen und gleichzeitig auch bei hohen Fermentaktivitäten mit ausreichender Substratkonzentration zu arbeiten. Wegen der schlechten Wasserlöslichkeit des Substrates wurde eine methanolische Lösung verwendet. Die Methanolkonzentration des Inkubationsansatzes (10\%) beeinflußt die Fermentaktivität nicht.

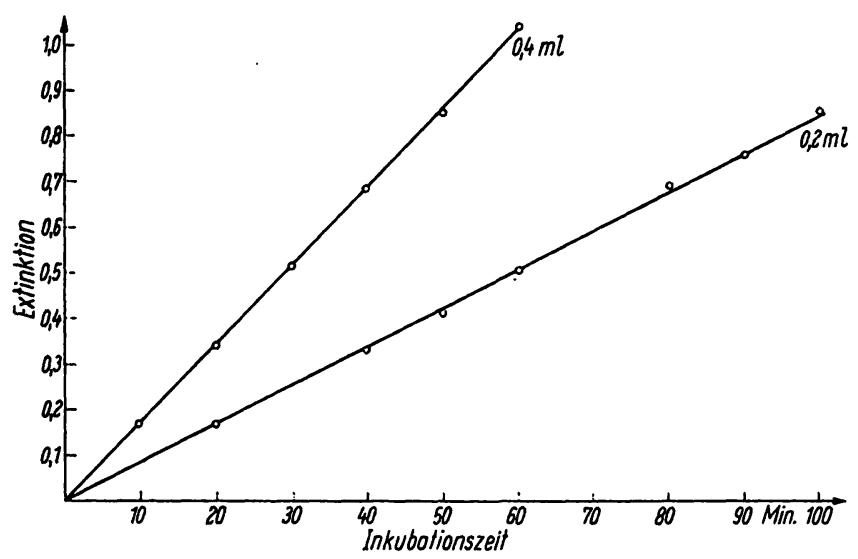

Abb. 4

Zeitabhängigkeit des Substratumsatzes mit verschiedenen Enzymaktivitäten (Serummenengen 0,4 und 0,2 ml). Meßtechn. Daten s. Abbildung 1

\section{Definition der LAP-Aktivität:}

1 Internationale LAP-Einheit entspricht dem Umsatz von $1 \mu \mathrm{Mol}$ Substrat $(=1 \mu \mathrm{Mol}$ freigesetztes $\beta$-Naphthylamin) $/ \mathrm{Min} . / 1000 \mathrm{~m} l$ Serum bei $37^{\circ}, 400 \mu \mathrm{g}$ Substratkonzentration im Ansatz und Trispuffer $0,05 \mathrm{~m}, \mathrm{pH}=7,2$. - Berechnung: $\frac{\mathrm{E}_{\mathrm{meB}}}{\mathrm{f}}=\mu \mathrm{g} \beta$-Naphthy!amin pro Ansatz $\times 7=\mathrm{IE}$. ( $(\mathrm{f}=$ Extinktion von $1 \mu \mathrm{g} \quad \beta$ Naphthylamin pro Ansatz (hier: (,0675)). Umrechnungsfaktor: $\frac{\mathrm{LAP}-\mathrm{E}}{30}=\mathrm{IE}$. IE $\times 30=\mathrm{LA}$ ' E: iheiten.

Eine LAP-Einheit ist die Menge $\beta$-Naphthylamin (in $\mu \mathrm{Mol})$, die von 1 Liter Serum bei einer Wasserbadtemperatur von $37^{\circ}$ und $30 \mathrm{Min}$. Inkubationsdauer freigesetzt wird.

Methodik und Normalwerte der übrigen Bestimmungen der Fermentaktivitäten

Die Serumfermentbestimmungen der GPT und GOT wurden im optischen Test nach W WRBURG durchgeführt. Die Aktivitätsangaben entsprechen den internationalen Einheiten (IE). $1 \mathrm{IE}$ ist gleich $1 \mathrm{Mol}$ Substratumsatz/ Min./1000 $\mathrm{ml}$ Serum.

Normalwert GPT: bis $14 \mathrm{IE}(22)$.

Normalwert GOT: bis 15 IE (22).

Alkalische Phosphate nach Huggins-Talaley,

Normalwerte: bis $15,0 \mathrm{HE}$.

(Einzelheiten vgl. Originalarbeiten.) Die Nüchternseren wurden am gleichen Tage aufgearbeitet, nur in Ausnahmefällen erst 24Stunden nach der Blutentnahme (Aufbewahrung bei $4^{\circ}$ ).

\section{Ergebnisse und Diskussion}

Die Normalwerte der LAP.Aktivität wurden an 40 männlichen und weiblichen Blutspenderseren unserer Klinik bestimmt. Der Normalwert bei Frauen liegt um 5 Einheiten unter dem der Männer. Im Einzelnen ergab das arithmetische Mittel für Männer einen Durchschnittswert von $27,7 \mathrm{E}$ mit einer Standardabweichung von $\pm 9,0 \mathrm{E}$, für Frauen: $22,7 \pm 6,4 \mathrm{E}$. Wir legen unseren 
Untersuchungen demnach folgende Normmerte zugrunde:

$$
\begin{aligned}
& \text { Männcr: } 18,6-36,7 \mathrm{IE}, \\
& \text { Fraucn: } 16,3-29,2 \mathrm{IE} .
\end{aligned}
$$

Um einen Überblick zu erhalten, welche Krankheiten mit einer LAP-Erhöhung einhergehen, und in welchem Zusammenhang sie zueinander stehen, wurde an 350 Patientenseren folgende Einteilung vorgenommen:

$\begin{array}{lll}\text { Männer } & & \text { Frallen } \\ \text { unter 18,6 IE } & \text { 1. Gruppe } & \text { unter 16,3 IE } \\ \text { 18,7-36,7 IE } & \text { 2. Gruppe } & \text { 16,4-29,2 IE } \\ \text { 36,8-39,5 IE } & \text { 3. Gruppe } & 29,3-36,7 \mathrm{IE} \\ \text { 39,6-69,0 IE } & \text { 4. Gruppe } & 36,8-63,9 \mathrm{IE} \\ \text { über 69,1 IE } & \text { 5. Gruppe } & \text { über 64,0 IE }\end{array}$

Zunächst trennen wir die Krankheitsbilder unterhalb des Normbereichs von 18,6 IE (क 16,3 IE) ab. Es folgt die 2. Gruppe mit dem Normbereich (18,7-36,7 und 16,4-29,2 IE). Die 3. Gruppe hielten wir bewußt eng begrenzt (2fache Standardabweichung), um die knapp oberhalb des Normbereichs auftretenden Krankheiten besser von den sicher pathologischen Werten der Gruppen 4 und 5 abgrenzen zu können. - In den folgenden Tabellen wurden die Krankheiten, ihrer Häufigkeit nach, in die einzelnen Rubriken eingeordnet, wobei wir von einer prozentualen Aufteilung absahen:

$\begin{array}{ll}\text { 1. Gruppe }(<16,3 ; \text { interer Normbereich) }) \\ \text { Asthma bronchiale } & \text { Rectum }-\mathrm{Ca} \\ \text { Thrombose } & \text { Mamma }-\mathrm{Ca} \\ \text { Herzinfarkt } & \text { Bronchitis } \\ \text { Ulcus pepticum } & \text { Epilepsic } \\ \text { Neuralgie } & \text { latente Tetanie }\end{array}$

2. Gruppe (Normalbereicl)):

chron. Hepatitis

Fettleber

Nephrose

Graviditas mens II

Lebercirrhose

Herzinfarkt

Bleivergiftung

Bronchial - $\mathrm{Ca}$

Diabetes mellitus
vegctative Dystonie
Anaemie, ak. und chron. Leukämie
Morbus Cushing
Hyperthyreose
Myxoedem
chron. Pankreatitis
Akromegalie

Von den von uns bevorzugt untersuchten Leber-, Galleund Pankreaskrankheiten finden wir im Normbereich vor allem die Diagnosen chron. Hepatitis und chron. Pankreatitis, aber auch Fettleber und Lebercirrhose vertreten. Diese Werte bestätigen auch andere Autoren $(4,3,14)$. - Die aufgeführten Blutkränkheiten ließen in keinem Falle eine Streuung außerhalb der Normgrenzen erkennen (15). Inwieweit Intoxikationen mit einer Steigerung der LAP-Aktivität einhergehen, vermögen wir auf Grund des zu geringen Untersuchungsgutes nicht zu beurteilen. Bei einem männlichen Pat. mit Bleiintoxikation fanden wir trotz mehrmaliger Be- stimmung keine Veränderungen im Serum. - Störungen des endokrinen Systems (Akromegalie, M. Cushing, Myxoedem, Hyperthyreose, Diabetes mellitus) verhalten sich unauffällig. Bei 2 Hyperthyreosefällen ragten die Werte noch in die Gruppe 3 hinein, stiegen aber nie in pathologische Aktivitätsbereiche auf. Auch konnten wir im Gegensatz zu amerikanischen Untersuchern (5) bei 15 Zuckerkranken keine Aktivitätssteigerungen finden.

\section{Gruppe \\ Graviditas mens V \\ Lebercirrhose \\ chron. Hepatitis \\ Fettleber \\ chron. Pankreatitis \\ Nephritis \\ rẹzidivierende Infekte \\ septisches Fieber}

An neuen Krankheitsbildern tauchen in der 3. Gruppe nur die diagnostisch bedeutungslosen, mit Fieberschüben einhergehenden, rezidivierenden Infekte und das septische Fieber auf. Mit BRESSLER (5) stimmen wir überein, daß bei einer Graviditas mens V die Serum-LAPWerte knapp oberhalb der Norm liegen, während sie in den ersten Schwangerschaftsmonaten völlig normal sind. Wahrscheinlich hängt diese Zunahme mit einer allgemeinen Fermentaktivitätserhöhung während der Schwangerschaft zusammen. Von den oben schon aufgeführten Leberkrankheiten und der chron. Pankreatitis finden wir noch Spitzenwerte in dieser Gruppe, die sicher pathologischen Grenzen bei 39,5 E (bzw. 36,7 E o) werden aber in keinem Fall überschritten.

\section{Gruppe: \\ Lebermetastasen \\ Cholangitis}

Bis auf 3 Ausnahmen finden wir nur Erkrankungen der Gałlenblase und Gallenwege. Die LAP-Werte zu. Ende der Schwangerschaft fielen ausnahmslos in diese Rubrik. Bei Niereninsuffizienz, sowie den nachzutragenden Ergebnissen bei Nephritis und Pyelonephritis aus der 3. Gruppe war stets eine Aktjvitätserhöhung vorhanden, über den diagnostischen Wert vermögen wir allerdings nichts auszusagen. Mit Goldbarg (16) sind wir der Meinung, daß die meisten entzündlichen Vorgänge mit leicht erhöhten LAP-Werten einhergehen. - Der Spitzenwert von 63,9 E wird bei Cholangitis und Cholecystitis nur in ganz wenigen Fällen erreicht. Diese Feststellung treffen auch andere Autoren $(4,5,2)$. während GöGGĖ (3) bei seinen klinisch und histologisch gesicherten Diagnosen vor allem bei Cholangitis Fermentwerte des 3fachen Normbereichs angibt. Bei unseren Patienten mit Gallen- und Gallenwegserkrankungen wurden derart hohe Aktivitäten nie gemessen. Sämtliche von uns untersuchten Pat. mit Malignomen und Lebermetastasen zeigten Werte zwischen 54,6 und 69,0 E. Damit ist eine exakte Abgrenzung gegenüber den Hochpathologischen der Gruppe 5 möglich, eine Ausnahme bildet nur ein Fall von Pankreas - Ca mit einer Fermenterhöhung auf über 147 IE. 


\section{Gruppe:}

Verschlußikterus akute Pankreatitis akute Hepatitis Pankreas - $\mathrm{Ca}$

Eine weitere Differenzierung des Okklusionsikterus (extrahepatisch, intrahepatisch) ist mit Hilfe der LAP nicht möglich. Ebensowenig vermögen wir auf Grund unserer Ergebnisse - im Gegensatz zu anderen (4, 5, 17) - nicht zu entscheiden, ob das Pankreas-Kopf-Ca (über 147 IE) oder das Pankreas-Körper-Ca die höchste LAP-Aktivität aufweist. Einleuchtend ist allerdings die vertretene Meinung $(5,7)$, daß dem Pankreas-Kopf-Ca infolge Stenosierung der Papilla Vateri und frühzeitiger Metastasierung die höchsten Aktivitätswerte zuzuschreiben sind, während die Diagnose des PankreasKörper-Ca erst auf dem Wege über die allgemeine Lebermetastasierung (bei uns zwischen 49,3 und 69,0 IE) zu klären ist. Die LAP-Werte bei akuter Pankreatitis und akuter Hepatitis liegen in unseren Ergebnissen zwischen 69,0 und 147 IE. So besteht eventuell die Möglichkeit der Abgrenzung zwischen Pankreatitis und Pankreas-Kopf-Ca, sowie zwischen Verschlußikterus (bis 137,7 IE) und Lebermetastasen $(49,4$ bis $69,0 \mathrm{IE})$ auf der einen und Cholangitis, Cholecystitis (39,5-69,0 IE) auf der anderen Seite.

Wenn auch die im Tierversuch ermittelte Aktivitätsverteilung der LAP (Tabelle 1) in den verschiedenen Organen anzeigt, daß eine organspezifische Aktivitätserhöhung im Serum nicht zu erwarten ist, so überraschen die in den Gruppen 4 und 5 gefundenen Diagnosen $(18,10)$.

Tab. 1

Aktivitätsverteilung der LAP in den verschiedenen tierischen Organen pro $100 \mathrm{mg}$ Gesamteiweiß

\begin{tabular}{lr}
\hline Speiseröhre & $11,4 \mathrm{mg}$ \\
Magenschleimhaut & $4,4 \mathrm{mg}$ \\
Magenmuskulatur & $11,7 \mathrm{mg}$ \\
Duodenum & $11,0 \mathrm{mg}$ \\
Jejunum & $27,1 \mathrm{mg}$ \\
Ileum & $22,6 \mathrm{mg}$ \\
Colon & $16,9 \mathrm{mg}$ \\
Leber & $9,1 \mathrm{mg}$ \\
Milz & $18,8 \mathrm{mg}$ \\
Niere & $22,5 \mathrm{mg}$ \\
Pankreas & $6,5 \mathrm{mg}$ \\
Herz & $6,3 \mathrm{mg}$ \\
Lunge & $9,2 \mathrm{mg}$ \\
\hline
\end{tabular}

- Es handelt sich bis auf 2 Ausnahmen (Niereninsuffizienz und Gravidität) um Erkrankungen der Leber, des Gallenwegsystems und des Pankreas. Wenn auch die akute Hepatitis epidemica mittels der Transaminasen relativ sicher diagnostiziert werden kann, so bleibt die Differentialdiagnose des Verschlußsyndroms immer noch schwierig, und ist auch durch Hinzuziehung von alkalischer Phosphatase und klinischer Symptomatik häufig nicht zu klären. - Ähnlich unsichere Laborato- riumsbefunde sind bei der akuten Pankreatitis bekannt, wenn auch der Amylaseaktivität im Serum und Urin eine gewisse Bedeutung zukommt (19), so daß ein Ferment, das bei den Erkrankungen im „Wetterwinkel des Oberbauches" (Leber, Galle, Pankreas) hohe Ausschläge liefert, ein willkommenes diagnostisches Hilfsmittel wäre.

In 23 Fällen, bei denen die LAP-Extinktionswerte pathologisch erhöht waren (s. o., Gruppe 4 und 5), bestimmten wir gleichzeitig die GOT und die GPT mit (Tabelle 2).

Tab. 2

\begin{tabular}{|c|c|c|c|c|c|}
\hline Nr. & Geschlecht & LAP & GPT & GOT & Diagnose \\
\hline 1. & w & 140 & 325 & 87 & akute Hepatitis \\
\hline 2. & $\mathrm{~m}$ & 139 & 72 & 54 & akute Pankrcatitis \\
\hline 3. & $\mathrm{~m}$ & 135 & 304 & 104 & akute Hepatitis \\
\hline 4. & w & 123 & 164 & 52 & Verschlußikterus \\
\hline 5. & w & 105 & 275 & 66 & akute Hepatitis \\
\hline 6. & $\mathrm{~m}$ & 102 & 49 & 15 & akute Pankreatitis \\
\hline$T$. & $\mathrm{m}$ & 102 & 38 & 23 & akute Pankreatitis \\
\hline 8. & $\mathrm{~m}$ & 99,5 & 45 & 18 & Verschlußikterus \\
\hline 9. & $\mathbf{w}$ & 97,2 & 64 & 32 & Verschlußikterus \\
\hline 10. & $\mathbf{w}$ & 93,1 & 254 & 91 & akute Hepatitis \\
\hline 11. & $\mathrm{~m}$ & 82,1 & 25 & 11 & akute Pankreatitis \\
\hline 12. & $\mathrm{~m}$ & 72 & 28 & 17 & akute Hepatitis \\
\hline 13. & w & 65,5 & 0 & 18 & akute Hepatitis \\
\hline 14. & $\mathrm{w}$ & 58,6 & 11 & 9 & Lebermetastasen \\
\hline 15. & $\mathrm{~m}$ & 56,5 & 17 & 20 & Lebermetastasen \\
\hline 16. & $\mathrm{w}$ & 56,3 & 18 & 25 & Lebermetastasen \\
\hline 17. & $\mathrm{w}$ & 56,1 & 127 & 152 & Cholecystitis \\
\hline 18. & $\mathbf{w}$ & 56,1 & 78 & 10 & Cholecystitis \\
\hline 19. & $\mathbf{w}$ & 54,7 & 124 & 95 & Cholecystitis \\
\hline 20. & $\mathrm{~m}$ & 54,3 & 163 & 65 & Lebermetastasen \\
\hline 21. & $\mathrm{~m}$ & 47,8 & 9 & 14 & Cholecystitis \\
\hline 22. & $\mathrm{~m}$ & 44,6 & 29 & 52 & Cholecystitis \\
\hline 23. & $\mathrm{w}$ & 36,5 & 14 & 18 & Lebermetastasen \\
\hline
\end{tabular}

Bisher wurde die Meinung vertreten, daß die GPT das empfindlichste Ferment zur Diagnostik der akuten Hepatitis ist $(20,21)$. Beweisend soll allerdings erst ihr Anstieg auf über $300 \mathrm{IE}$ sein. Vergleichend mit unseren Ergebnissen ergibt sich in 4 von 6 Fällen eine GPTErhöhung auf über $250 \mathrm{IE}$, davon 2 mal über $300 \mathrm{IE}$. Die beiden restlichen Untersuchungen zeigen bei klinisch gesicherter Diagnose hochpathologische LAPWerte gegenüber der GPT, die im Normbereich liegt. Ein weitaus geringerer Aussagewert kommt der GOT zu, die ebenfalls in 4 Fällen erhöht ist, aber 100 IE nicht überschreitet, während die LAP bis $140 \mathrm{IE}$ ansteigt. Bei akuter Pankreatitis liegt die Spitze der GPT bei 72 IE und ist in allen Fällen erhöht. Die GOT zeigt 2 völlig normale Werte, die LAP-Aktivität liegt im Gruppenbereich V (über 69,0 IE). Der Verschlußikterus zeigt LAP-Werte, die nur knapp unter 99 IE liegen, die GOT fällt genau wie die GPT aus den Normbereich heraus, aber in keinem Falle könnte man hier eine differential- 
diagnostische Aussage treffen. - Noch weniger Differenzierungsmöglichkeit bietet sich bei Lebermetastasen. Hier liegen die LAP-Werte zwischen 49,3 und 69,0 IE, die GPT schwankt unsicher zwischen 163 und 4 IE, die GOT hat einen kleineren Ausfallbezirk von 9 bis 65 IE. In 5 Fällen von Cholangitis und Cholecystitis sind die Transaminasen dagegen stärker erhöht, als es in den vorhergehenden Krankheitsbildern der Fall war. GöGGEL und SHAY bestätigen allerdings in ihren Arbeiten, daB etwa $1 / 3$ der Transaminasewerte den Normbereich nicht verlassen $(3,14)$.

Eine besondere Bedeutung der LAP ergibt sich aus dem Vergleich mit der alkalischen Phosphatase. Wir prüften die Verhaltensweise von 23 Seren unter besonderer Berücksichtigung des Verschlußikterus. Wie aus der Tabelle 3 ersichtlich ist, geht der Verschlußikterus mit erhöhten LAP- und Phosphatasewerten einher. Darüber hinaus steigt die alkalische Phosphatase auch bei der akuten Hepatitis und den Lebermetastasen verschieden stark an. Ob allerdings beim Verschlußikterus eine strenge Parallelität (3) beider Enzyme zueinander besteht, vermögen wir auf Grund des noch zu geringen Untersuchungsgutes nicht zu entscheiden. Keine Phosphataseerhöhung finden wir bei der chron. Hepatitis und der Cholangitis.
Tab. 3

\begin{tabular}{|c|c|c|c|c|}
\hline Nr. & Geschlecht & LAP & alk. Ph. & Diagnose \\
\hline 1. & $\mathrm{~m}$ & 250 & 10 & Pankreas - Ca \\
\hline 2. & $w$ & 144 & 41 & Verschlußikterus \\
\hline 3. & w & 103 & 7 & akute Hepatitis \\
\hline 4. & $\mathrm{~m}$ & 102 & 54 & Verschlußikterus \\
\hline 5. & $\mathrm{~m}$ & 101 & 87 & Verschlußikterus \\
\hline 6. & $\mathrm{w}$ & 78,5 & 28 & akute Hepatitis \\
\hline 7. & $\mathrm{~m}$ & 72 & 19 & akute Hepatitis \\
\hline 8. & $\mathrm{~m}$ & 70,7 & 50 & Lebermetastasen \\
\hline 9. & $\mathrm{~m}$ & 70 & 6 & akute Hepatitis \\
\hline 10. & $w$ & 69 & 39 & Lebermetastasen \\
\hline 11. & $w$ & 68,4 & 6 & Lebermetastasen \\
\hline 12. & $\mathbf{w}$ & 58,8 & 15 & Lebermetastasen \\
\hline 13. & $\mathrm{w}$ & 56 & 13 & Lebermetastasen \\
\hline 14. & $\mathrm{~m}$ & 54 & 14 & Lebermetastasen \\
\hline 15. & $\mathrm{w}$ & 53,8 & 30 & Lebermetastasen \\
\hline 16. & $\mathrm{~m}$ & 53,8 & 12 & Lebermetastasen \\
\hline 17. & $\mathrm{~m}$ & 47,8 & 13 & Cholecystitis \\
\hline 18. & w & 46,6 & 7 & Cholecystitis \\
\hline 19. & $\mathrm{~m}$ & 45 & 15 & Cholecystitis \\
\hline 20. & w & 45 & 10 & Cholecystitis \\
\hline 21. & $\mathrm{~m}$ & 44,6 & 16 & Lebermetastasen \\
\hline 22. & $\mathrm{~m}$ & 40,7 & 8 & Cholecystitis \\
\hline 23. & $\mathrm{w}$ & 39,6 & 9 & chronische Hepatitis \\
\hline
\end{tabular}

\section{Literatur}

1. Linderstøm-Lang, K., Hoppe-Seyler's Z. physiol. Chem. 182, 151 (1929). - 2. MiLLER, A. L. und L. WorsLey, Brit. Med. J. 12, 1419 (1960). - 3. Göggel, K. H., W. CReutzFeldt und J. MurUCaS, Dtsch. med. Wschr. 85, 1756 und 1808 (1960). - 4. Banks, B. M., E. P. Pineda, J. A. Goldbarg und A. M. Rutenberg, N. England J. Med. 263, 1277 (1960). - 5. BressLeR, R., B. R. Forsyth und G. Klarskin, J. Laborat. Clin. Med., S. Louis 56, 417 (1960). - 6. Goldbarg, J. A. und A. M. Rutenberg, Cancer 11, 283 (1958). - 7. Pineda, E. P., J. A. Goldbarg und A. M. Rutenberg, Surgical Forum 249 (1960). - 8. SMrth, E. L. und D. H. SpackmanN, J. biol. Chemistry 217, 271 (1955). 9. Braun-Falco, O. und K. Salfeldt, Arch. klin. exp. Dermatol. 205, 103 (1957). - 10. SCHÖN, H., B. RäsSLER, J. Rico-IrLeS und N. Hennnag, Verteilung der LAP im tierischen Organismus (In Vorbereitung). - 11. Green, M. N., Kwan-Chung Tsou, R. Bressler und A. M. SeligmanN, Arch. Biochemistry 57, 458
(1955). - 12. Braun-Falco, O. und K. Salfeldt, Arch. klin. exp. Dermatol. 204, 407 (1957). - 13. Folk, J. E. und M. S. BurSTONE, Proc., Soc. exp. Biol. Med. 89, 473 (1955). - 14. Shay, H., D. Sun und H. Siplet, Amer. J. Digest Dis. 5, 217 (1960). 15. Rutenberg, A. M., J.A. Goldbarg und E. P. Pineda, N. England J. Med. 259, 469 (1958). - 16. Goldbarg, J. A., E. P. Pineda und A. M. Rutenderg, Amer. J. Clin. Path. 32, 571 (1959). - 17. Pineda, E. P., J. A. Goldbarg, J. A. Banks und A. M.: Rutenderg, Gastroenterology, Baltimore 38, 698 (1960). 18. Richterich, R., Enzymopathologie, Springer-Verlag, BerlinGöttingen-Heidelberg (1958). - 19. HeINKEL, K. und W. LAr, Ärztl. Laborat. 2, 82 (1956). - 20. ScHÖN, H., B. ENGLISCH und H. Wüst, Dtsch. mẹ. Wschr. 85, 265 (1960). - 21. ScнöN, H. und H. Wüst, Dtsch. med. Wschr. 86, 281 (1961). - 22. LEIPOLD, H., H. Wüst und H. SснӧN, Dtsch. med. Wschr. 41 (1961).

Dozent Dr. H. Schön Medizinische Universitäts-Klinik 852 Erlangen, Krankenhausstr. 12 